\title{
Priority queueing with finite buffer size and randomized push-out mechanism
}

Konstantin E. Avrachenkov — Nikita O. Vilchevsky — Georgy L. Shevlyakov

$\mathbf{N}^{\circ} 4434$

Mars 2002

THÈME 1 



\title{
Priority queueing with finite buffer size and randomized push-out mechanism
}

\author{
Konstantin E. Avrachenkov* ${ }^{*}$ Nikita O. Vilchevsky ${ }^{\dagger}$, Georgy L. Shevlyakov ${ }^{\ddagger}$ \\ Thème 1 - Réseaux et systèmes \\ Projet MISTRAL
}

Rapport de recherche $\mathrm{n}^{\circ} 4434$ - Mars 2002 - 17 pages

\begin{abstract}
The non-preemptive priority queueing with a finite buffer is considered. We introduce a randomized push-out buffer management mechanism which allows to control very efficiently the loss probability of priority packets. The packet loss probabilities for priority and non-priority traffic are calculated using the generating function approach. For the particular case of the standard non-randomized push-out scheme we obtain explicit analytic expressions. The theoretical results are illustrated by a numerical example.
\end{abstract}

Key-words: priority queueing, finite buffer, randomized push-out

\footnotetext{
* INRIA Sophia Antipolis, 2004 Route des Lucioles, B.P.93, 06902, France, e-mail: k.avrachenkov@sophia.inria.fr

$\dagger$ St. Petersburg State Technical University, Polytechnicheskaya, 29, St.Petersburg, 195251, Russia, e-mail: vilch@kit.amd.stu.neva.ru

‡ St. Petersburg State Technical University, Polytechnicheskaya, 29, St.Petersburg, 195251, Russia, e-mail: shev@stat.hop.stu.neva.ru
} 


\section{La file d'attente prioritaire à capacité limitée avec push-out stochastique}

Résumé : Nous étudions la file d'attente prioritaire (non-preemptive) à capacité limitée. Nous introduisons un mécanisme de push-out stochastique. Avec ce mécanisme la perte de paquets prioritaires peut être contrôlée de manière simple et efficace. Pour résoudre le modèle, nous utilisons la méthode de la fonction génératrice. En cas particulier du mécanisme de push-out non-stochastique nous avons réussi à obtenir des formules analytiques explicites. Les résultats théoriques sont illustrés par un exemple numérique.

Mots-clés : file d'attente prioritaire, capacité limitée, push-out stochastique 


\section{Introduction}

Priority queueing discipline has a number of important applications in telecommunications and computer networks, e.g., Differentiated Services architecture for the Internet [7].

Here we consider the non-preemptive priority queueing system with two classes of packets. Class 1 packets have priority over class 2 packets. The packets of class 1 (2) arrive into the buffer according to the Poisson process with rate $\lambda_{1}\left(\lambda_{2}\right.$, resp.). The service time has the exponential distribution with the same rate $\mu$ for each class. The service times are independent of the arrival processes. The buffer has a finite size $N$ and it is shared by both types of customers. If the buffer is full, a new coming customer of class 1 can push out of the buffer a customer of class 2 with the probability $\alpha$. Note that if $\alpha=1$ we retrieve the standard non-randomized push-out mechanism.

The infinite buffer priority queueing has been studied thoroughly in the past $[4,8,9]$. The case of finite buffer priority queueing received considerably less attention. Kapadia et al $[5,6]$ analyzed the $\mathrm{M} / \mathrm{M} / \mathrm{s} / \mathrm{K}$ type finite buffer non-preemptive priority queueing with non-randomized push-out mechanism. Bondi [1] analyzed the $\mathrm{M} / \mathrm{M} / 1 / \mathrm{K}$ type preemptive and non-preemptive priority queueing with the following buffer management schemes: complete partitioning, complete sharing and sharing with minimum allocation. Wagner and Krieger [10] analyzed the $M / M / 1 / K$ type non-preemptive priority queueing with the complete sharing buffer management scheme and with the class-dependent service rates. In [2] Cheng and Akyildiz considered the priority queueing with general service time distributions and a general service discipline function. They analyzed the push-out with threshold as the buffer management scheme.

Most of the above works use recursive relations to solve steady state Kolmogorov equations. We use the generating function based approach, which only requires the solution of a linear system of $N$ equations. To our best knowledge, the randomized push-out mechanism is analyzed for the first time. In particular, we show that with the randomized push-out scheme it is easy to control the loss probability of priority packets in a very large range. Furthermore, in the particular case of non-randomized push-out we obtain an explicit analytic expressions for the loss probabilities that are simpler than expressions in Kapadia et al [5,6]. Finally we conclude the paper with a numerical example.

$\mathrm{RR} \mathrm{n}^{\circ} 4434$ 


\section{Main results}

Let us denote by $p(i, n)$ the stationary probability of the event that there are $n$ packets in the queue including $i$ packets of class 1 . We also denote by $p_{0}$ the stationary probability of the event that there are no packets in the system. These probabilities satisfy the following stationary Kolmogorov equations:

$$
\left(\lambda_{1}+\lambda_{2}\right) p_{0}=\mu p(0,0)
$$

- $n=0$

$$
\left(\lambda_{1}+\lambda_{2}+\mu\right) p(0,0)=\mu p(1,1)+\mu p(0,1)+\left(\lambda_{1}+\lambda_{2}\right) p_{0}
$$

- $0<n<N$

$$
\begin{aligned}
& \left(\lambda_{1}+\lambda_{2}+\mu\right) p(0, n)=\quad \mu p(1, n+1)+\mu p(0, n+1) \quad+\lambda_{2} p(0, n-1), \\
& \left(\lambda_{1}+\lambda_{2}+\mu\right) p(i, n)=\mu p(i+1, n+1) \quad+\lambda_{1} p(i-1, n-1) \quad+\lambda_{2} p(i, n-1), \\
& \left(\lambda_{1}+\lambda_{2}+\mu\right) p(i, n)=\mu p(n+1, n+1) \quad+\lambda_{1} p(n-1, n-1)
\end{aligned}
$$

- $n=N$

$$
\begin{array}{rrrrr}
\left(\alpha \lambda_{1}+\mu\right) p(0, N) & = & \lambda_{2} p(0, N-1), & \\
\left(\alpha \lambda_{1}+\mu\right) p(i, N) & = & \lambda_{1} p(i-1, N-1) & +\lambda_{2} p(i, N-1) & +\alpha \lambda_{1} p(i-1, N), \\
\mu p(N, N) & = & \lambda_{1} p(N-1, N-1) & & +\alpha \lambda_{1} p(N-1, N) .
\end{array}
$$

Next we introduce the generating function for $p(i, n)$ by the index $i$

$$
F_{n}(x)=\sum_{i=0}^{n} p(i, n) x^{i} .
$$

Using the above given Kolmogorov equations, we obtain relations for the generating functions $F_{n}(x), n=0,1, \ldots, N$ :

- $n=0$

$$
\left(\lambda_{1}+\lambda_{2}+\mu\right) F_{0}(x)=\frac{\mu}{x}\left[F_{1}(x)-p(0,1)\right]+\mu p(0,1)+\left(\lambda_{1}+\lambda_{2}\right) p_{0},
$$

- $0<n<N$

$\left(\lambda_{1}+\lambda_{2}+\mu\right) F_{n}(x)=\frac{\mu}{x}\left[F_{n+1}(x)-p(0, n+1)\right]+\mu p(0, n+1)+\left(\lambda_{1} x+\lambda_{2}\right) F_{n-1}(x)$.

In particular, we get the following boundary condition 
- $n=N$

$\left(\alpha \lambda_{1}+\mu\right) F_{N}(x)-\alpha \lambda_{1} p(N, N) x^{N}=\left(\lambda_{1} x+\lambda_{2}\right) F_{N-1}(x)+\alpha \lambda_{1} x F_{N}(x)-\alpha \lambda_{1} x^{N+1} p(N, N)$.

Let us now introduce the generating function for $F_{n}(x)$ by the index $n$

$$
\Phi(x, y)=\sum_{n=0}^{N-1} F_{n}(x) y^{n} .
$$

The generating function $\Phi(x, y)$ satisfies equation (2) given in Lemma 1 below.

Lemma 1 The generating function $\Phi(x, y)$ satisfies the following equation

$$
\begin{gathered}
{\left[(\rho+1) x y-x y^{2}\left(\rho_{1} x+\rho_{2}\right)-1\right] \Phi(x, y)=-y^{N+1} x\left(\rho_{1} x+\rho_{2}\right) F_{N-1}(x)+y^{N} F_{N}(x)} \\
+y(x-1) A(y)+(x y-1) \rho p_{0}, \\
\text { where } \rho_{i}=\lambda_{i} / \mu, \rho=\rho_{1}+\rho_{2} \text { and } A(y)=\sum_{n=0}^{N-1} p(0, n+1) y^{n} .
\end{gathered}
$$

In the next theorem we determine the generating function $\Phi(x, y)$.

Theorem 1 The generating function $\Phi(x, y)$ is given by

$$
\begin{aligned}
\Phi(x, y)= & \frac{\left[1-x y+\alpha \rho_{1} x y(x-1)\right] y^{N} V_{N-1}(x)+y(x-1) A(y)}{(\rho+1) x y-x y^{2}\left(\rho_{1} x+\rho_{2}\right)-1} \\
& +\frac{[1-x y] x^{N} y^{N} p(N, N)+\rho[x y-1] p_{0}}{(\rho+1) x y-x y^{2}\left(\rho_{1} x+\rho_{2}\right)-1},
\end{aligned}
$$

where

$$
\begin{aligned}
V_{N-1}(x)= & \sum_{k=0}^{N-1} x^{k} p(k, N) \\
A(y)= & -\alpha \rho y^{N-1} p(0, N) \\
& +\sum_{k=1}^{N-1}\left[\rho_{2} y^{N-k} \frac{U_{k-1}(t)}{\rho_{1}^{(k+1) / 2}}-\alpha \rho y^{N-k-1} \frac{U_{k}(t)}{\rho_{1}^{k / 2}}+\alpha y^{N-k-1} \frac{U_{k-1}(t)}{\rho_{1}^{(k-1) / 2}}\right] p(k, N) \\
& +\rho_{2} \frac{U_{N-1}(t)}{\rho_{1}^{(N+1) / 2}} p(N, N)
\end{aligned}
$$

with $t=\left(\rho+1-\rho_{2} y\right) /\left(2 \rho_{1}^{1 / 2}\right)$ and where probabilities $p(k, N), k=0, \ldots, N$ can be obtained as a solution to the following system of linear equations

$\mathrm{RR} \mathrm{n}^{\circ} 4434$ 
- $s=0$

$$
\alpha \rho_{1} C_{N-1}^{1}\left(t_{0}\right) p(N-1, N)+\left[\rho C_{N-1}^{1}\left(t_{0}\right)-\rho_{1}{ }^{1 / 2} C_{N}^{1}\left(t_{0}\right)\right] p(N, N)+\rho \rho_{1}{ }^{(N+1) / 2} p_{0}=0
$$

- $0<s<N$

$$
\begin{gathered}
\sum_{k=0}^{s-1}\left[\rho \frac{C_{N-s-1}^{s-k}\left(t_{0}\right) \rho_{1}{ }^{k+1}}{\left(-\rho_{2}\right)^{k+1}}-\rho_{1}{ }^{3 / 2}(1+\alpha \rho) \frac{C_{N-s}^{s-k}\left(t_{0}\right) \rho_{1}{ }^{k}}{\left(-\rho_{2}\right)^{k+1}}\right. \\
\left.+\rho_{1} \alpha \frac{C_{N-s-1}^{s-k+1}\left(t_{0}\right) \rho_{1}{ }^{k}}{\left(-\rho_{2}\right)^{k}}\right] p(N-1-k, N)+\alpha \rho_{1}{ }^{s+1} \frac{C_{N-s-1}^{1}\left(t_{0}\right)}{\left(-\rho_{2}\right)^{s}} p(N-1-s, N) \\
+\left[\rho C_{N-s-1}^{s+1}\left(t_{0}\right)-\rho_{1}{ }^{3 / 2} C_{N-s}^{s+1}\left(t_{0}\right)\right] p(N, N)=0,
\end{gathered}
$$

- $s=N$

$$
-\rho_{1}{ }^{3 / 2}(1+\alpha \rho) \sum_{k=0}^{N-1} \frac{C_{0}^{N-k}\left(t_{0}\right) \rho_{1}{ }^{k}}{\left(-\rho_{2}\right)^{k+1}} p(N-1-k, N)-\rho_{1}{ }^{1 / 2} C_{0}^{N+1}\left(t_{0}\right) p(N, N)=0
$$

with $U_{n}(x)$ and $C_{n}^{\nu}(x)$ denoting the Chebyshev polynomials of the second kind and the Gegenbauer polynomials [3], respectively, and

$$
p_{0}=(1-\rho) /\left(1-\rho^{N+2}\right), \quad t_{0}=(\rho+1) /\left(2 \rho_{1}^{1 / 2}\right) .
$$

Proof: given in Appendix.

Once we know the value of $p(N, N)$, we can calculate the loss probabilities of class 1 and class 2 packets.

Theorem 2 The loss probabilities of class 1 and class 2 packets are given by the following formulae

$$
\begin{gathered}
P_{\text {loss }}^{(1)}=p(N, N)+(1-\alpha)\left[P_{N}-p(N, N)\right], \\
P_{\text {loss }}^{(2)}=P_{N}+\alpha \frac{\rho_{1}}{\rho_{2}}\left[P_{N}-p(N, N)\right]
\end{gathered}
$$

where

$$
P_{N}=\frac{1-\rho}{1-\rho^{N+2}} \rho^{N+1}
$$


Proof: A priority packet can be lost either when the whole buffer is filled only with priority packets or when there are some packets of class 2 but with probability $1-\alpha$ the push-out mechanism is not enabled. The probability of the first event is $p(N, N)$ and the probability of the second event is $\sum_{k=0}^{N-1} p(k, N)=P_{N}-p(N, N)$. Thus, we obtain formula (3).

The stream of lost packets of class 2 consists of the stream of packets with rate $\lambda_{2} P_{N}$ lost when the buffer is full and the stream of packets with rate $\alpha \lambda_{1}\left(P_{n}-\right.$ $p(N, N))$ pushed out by packets of class 1 . Since the system is ergodic, we obtain formula (4).

We note that if $\alpha=0$ (no push-out), the loss probabilities for two classes coincide and are equal to $P_{N}$. We also would like to note that due to the fact that the service time distribution is the same for the two classes, the expressions for $p_{0}, F_{N}(1)$ and $\Phi(1,1)$ could be obtained immediately by elementary considerations.

In the particular case of the non-randomized push-out mechanism, that is, when $\alpha=1$, we are able to calculate the loss probabilities explicitly.

Theorem 3 The loss probabilities of class 1 and class 2 packets in the case of nonrandomized push-out mechanism are given by

$$
\begin{gathered}
P_{\text {loss }}^{(1)}=\rho \rho_{1}^{N} \frac{\left(1-\rho_{1}\right)\left(1-\rho^{N+1}\right)}{\left(1-\rho_{1}^{N+1}\right)\left(1-\rho^{N+2}\right)}, \\
P_{\text {loss }}^{(2)}=P_{N}+\frac{\rho_{1}}{\rho_{2}}\left[P_{N}-P_{\text {loss }}^{(1)}\right] .
\end{gathered}
$$

Proof: In the case of non-randomized push-out mechanism $(\alpha=1)$, the equation for the generating function (2) takes the form

$$
\begin{gathered}
{\left[(\rho+1) x y-x y^{2}\left(\rho_{1} x+\rho_{2}\right)-1\right] \Phi(x, y)=y^{N}\left[1-x y+\rho_{1} x(x-1) y\right] F_{N}(x)} \\
+y(x-1) A(y)+\rho_{1}(1-x) x^{N+1} y^{N+1} p(N, N)+(x y-1) \rho p_{0} .
\end{gathered}
$$

Setting $x=1$ in (7), and then reducing it by the term $(y-1)$, we get

$$
(1-\rho y) \Phi(1, y)=\rho p_{0}-y^{N} F_{N}(1) \text {. }
$$

Then in the above equation we take subsequently $y=1$ and $y=1 / \rho$ to obtain

$$
(1-\rho) \Phi(1,1)=\rho p_{0}-F_{N}(1)
$$

$\mathrm{RR} \mathrm{n}^{\circ} 4434$ 
and

$$
0=\rho p_{0}-\frac{1}{\rho^{N}} F_{N}(1)
$$

Solving equations (8) and (9) together with the normalization condition

$$
\Phi(1,1)+p_{0}+F_{N}(1)=1,
$$

we obtain the following expressions for $p_{0}, F_{N}(1)$ and $\Phi(1,1)$ :

$$
p_{0}=\frac{1-\rho}{1-\rho^{N+2}}, \quad F_{N}(1)=\frac{1-\rho}{1-\rho^{N+2}} \rho^{N+1}, \quad \Phi(1,1)=\frac{1-\rho^{N+1}}{1-\rho^{N+2}} \rho .
$$

Next we take $y=1$ in equation (7) and then reduce it by the term $(x-1)$

$$
\left(1-\rho_{1} x\right) \Phi(x, 1)=-\left(1-\rho_{1} x\right) F_{N}(x)+A(1)-\rho_{1} x^{N+1} p(N, N)+\rho p_{0} .
$$

We now set subsequently $x=1$ and $x=1 / \rho_{1}$ in the above equation. This results in the following two equations:

$$
\begin{gathered}
\left(1-\rho_{1}\right) \Phi(1,1)=-\left(1-\rho_{1}\right) F_{N}(1)+A(1)-\rho_{1} p(N, N)+\rho p_{0} \\
0=A(1)-\frac{1}{\rho_{1}^{N}} p(N, N)+\rho p_{0} .
\end{gathered}
$$

Solving equations (10) and (11), we obtain

$$
p(N, N)=\frac{\left(1-\rho_{1}\right)\left(1-\rho^{N+1}\right)}{\left(1-\rho_{1}^{N+1}\right)\left(1-\rho^{N+2}\right)} \rho \rho_{1}^{N} .
$$

The loss probability of class 1 packets $P_{\text {loss }}^{(1)}$ is given by $p(N, N)$. Then, we note that the stream of lost packets of class 2 consists of the stream of packets with rate $\lambda_{2} F_{N}(1)$ lost when the buffer is full and the stream of packets with rate $\lambda_{1}\left(F_{N}(1)-\right.$ $p(N, N))$ pushed out by packets of class 1 . Hence, using the ergodicity property of the system, we obtain formula (6) for $P_{\text {loss }}^{(2)}$.

\section{$3 \quad$ Numerical Example and Conclusions}

In order to calculate the coefficients of the linear system for $p(i, N), i=0, \ldots, N$ in Theorem 1, we need to compute the Gegenbauer polynomials. We suggest to use the recursive formulae

$$
(n+1) C_{n+1}^{k}(t)=2(n+k) t C_{n}^{k}(t)-(n+2 k-1) C_{n-1}^{k}(t),
$$


with $C_{0}^{k}(t)=1$ and $C_{1}^{k}=2 k t[3, \mathrm{v} .2, \mathrm{p} .175]$. We note that the coefficient matrix has the following form

$$
\left[\begin{array}{ccccc}
0 & \cdots & 0 & * & * \\
\vdots & 0 & * & & \vdots \\
0 & * & & & \\
& & & & \vdots \\
* & \cdots & & \cdots & *
\end{array}\right] .
$$

This nearly triangular structure simplifies the solution of the linear system for $p(i, N), i=$ $0, \ldots, N$. To improve the conditioning of the linear system, we propose to use the normalization condition

$$
\sum_{k=0}^{N} p(k, N)=P_{N}
$$

in place of the last equation. As an example, we take the following values for the system parameters: $\rho_{1}=0.2, \rho_{2}=0.9$ and $N=30$. We consider a typical scenario when the intensity of arrival of higher priority packets is smaller than the intensity of arrival of lower priority packets. Once the system for $p(i, N), i=0, \ldots, N$ is solved, we can calculate packet loss probabilities by the formulae given in Theorem 2 . In Figure 1 we plot the packet loss probabilities for two classes as a function of parameter $\alpha$. In the particular cases, $\alpha=0$ and $\alpha=1$, we can calculate the loss probabilities using the explicit analytic formulae (the formula for $P_{N}$ and the formulae in Theorem 3). As one can see, the numerical solutions for $\alpha=0$ and $\alpha=1$ coincide with the explicit analytical solutions.

There are at least two important conclusions that we can draw from Figure 1. First, by changing parameter $\alpha$ we tune the loss probability of the priority packets in a very large range, that is from 0.0954 down to $8.54 \times 10^{-22}$. Then, we note that with the increase of $\alpha$ the loss of non-priority packets does not deteriorate as quickly as the acceptance of priority packets improves. Namely, the loss probability of the non-priority packets only changes by $22 \%$. Second, we note that the dependence of the packet loss probabilities for both classes on the parameter $\alpha$ is very close to linear. Thus, the randomized push-out mechanism can easily be applied for the engineering of the priority queueing systems.

$\mathrm{RR} \mathrm{n}^{\circ} 4434$ 


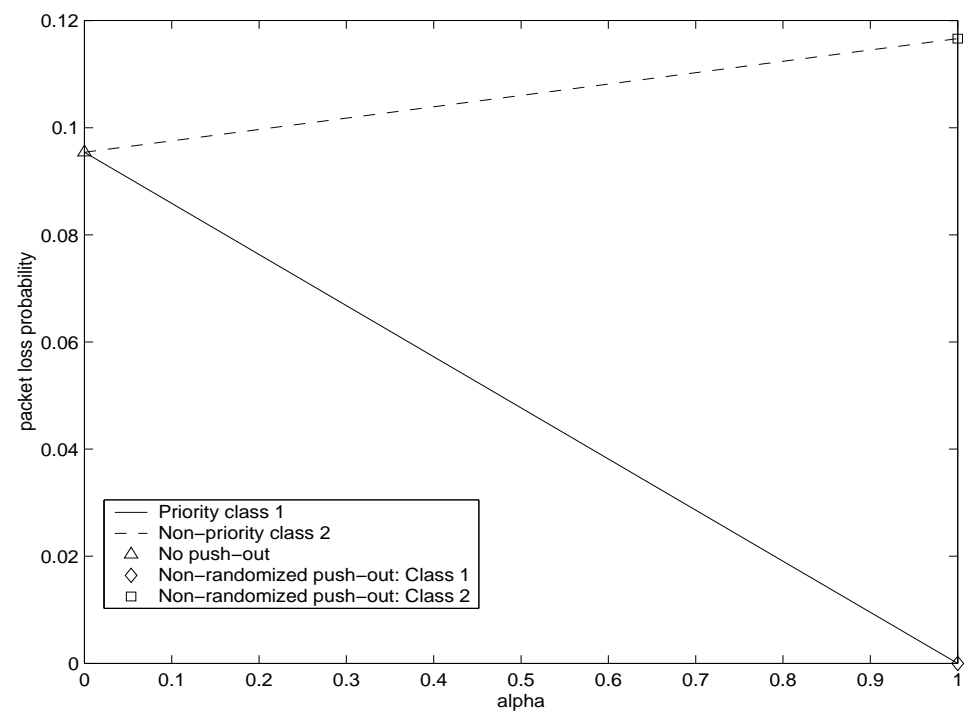

Figure 1: Numerical example with $\rho_{1}=0.2, \rho_{2}=0.9$ and $N=30$.

\section{Appendix. The proof of Theorem 1}

By substituting boundary condition (1) into equation (2) for the generating function $\Phi(x, y)$, we get

$$
\begin{gathered}
{\left[(\rho+1) x y-x y^{2}\left(\rho_{1} x+\rho_{2}\right)-1\right] \Phi(x, y)=\left[1-x y+\alpha \rho_{1} x y(x-1)\right] y^{N} V_{N-1}(x)} \\
+[1-x y] x^{N} y^{N} p(N, N)+y(x-1) A(y)+\rho[x y-1] p_{0}
\end{gathered}
$$

where $V_{N-1}(x)=\sum_{i=0}^{N-1} x^{i} p(i, N)$, and hence the expression for $\Phi(x, y)$.

Next, we set $z:=x y$ and rewrite equation (12) as follows:

$$
\begin{gathered}
{\left[\left(\rho_{1}+\rho_{2}+1\right) z-\rho_{1} z^{2}-\rho_{2} y z-1\right] \Phi\left(\frac{z}{y}, y\right)=\left[(1-z) y+\rho_{1} \alpha(z-y) z\right] y^{N-1} V_{N-1}\left(\frac{z}{y}\right)} \\
+(z-y) A(y)+(1-z) z^{N} p(N, N)+\rho(z-1) p_{0} .
\end{gathered}
$$

Let us now consider the analyticity condition for the generating function $\Phi(z / y, y)$. Namely, the following two conditions have to be satisfied simultaneously

$$
\left(\rho_{1}+\rho_{2}+1\right) z-\rho_{1} z^{2}-\rho_{2} y z-1=0,
$$


$\left[(1-z) y+\rho_{1} \alpha(z-y) z\right] y^{N-1} V_{N-1}\left(\frac{z}{y}\right)+(z-y) A(y)+(1-z) z^{N} P(N, N)+\rho(z-1) p_{0}=0$.

The first condition can be rewritten as

$$
\rho_{2}(y-z) z=(1-z)(\rho z-1)
$$

which gives

$$
y-z=\frac{(1-z)(\rho z-1)}{\rho_{2} z} .
$$

We substitute the above expression for $y-z$ into the first two terms of the second analyticity condition and then reduce it by $1-z$, to get

$$
\left(y-\frac{\rho_{1}}{\rho_{2}} \alpha(\rho z-1)\right) y^{N-1} V_{N-1}\left(\frac{z}{y}\right)-\frac{\rho z-1}{\rho_{2} z} A(y)+z^{N} p(N, N)-\left(\rho_{1}+\rho_{2}\right) p_{0}=0 .
$$

Next we denote by $a$ and $b$ the roots of the following quadratic equation with respect to the variable $z$

$$
\left(\rho_{1}+\rho_{2}+1\right) z-\rho_{1} z^{2}-\rho_{2} y z-1=0 .
$$

Now we substitute subsequently the roots $a$ and $b$ into (13), which allows us to eliminate $A(y)$

$$
\begin{gathered}
\frac{\rho b-1}{b}\left(y-\frac{\rho_{1}}{\rho_{2}} \alpha(\rho a-1)\right) y^{N-1} V_{N-1}\left(\frac{a}{y}\right)-\frac{\rho a-1}{a}\left(y-\frac{\rho_{1}}{\rho_{2}} \alpha(\rho b-1)\right) y^{N-1} V_{N-1}\left(\frac{b}{y}\right) \\
+\left(\frac{\rho b-1}{b} a^{N}-\frac{\rho a-1}{a} b^{N}\right) p(N, N)-\rho\left(\frac{\rho b-1}{b}-\frac{\rho a-1}{a}\right) p_{0}=0 .
\end{gathered}
$$

Taking into account the properties of roots of the quadratic equation

$$
a b=1 / \rho_{1}, \quad(\rho a-1)(\rho b-1)=\frac{\rho_{2}}{\rho_{1}}(\rho y-1),
$$

we have

$$
\begin{gathered}
\left(\left(\rho-\rho_{1} a\right) y-q(\rho y-1) \rho_{1} a\right) y^{N-1} V_{N-1}\left(\frac{a}{y}\right)-\left(\left(\rho-\rho_{1} b\right) y-q(\rho y-1) \rho_{1} b\right) y^{N-1} V_{N-1}\left(\frac{a}{y}\right) \\
+\left(\rho\left(a^{N}-b^{N}\right)-\rho_{1}\left(a^{N+1}-b^{N+1}\right)\right) p(N, N)+\rho \rho_{1}(a-b) p_{0}=0 \\
\rho y^{N}\left(V_{N-1}\left(\frac{a}{y}\right)-V_{N-1}\left(\frac{b}{y}\right)\right)-\rho_{1}(y+q(\rho y-1))\left(a V_{N-1}\left(\frac{a}{y}\right)-b V_{N-1}\left(\frac{b}{y}\right)\right) y^{N-1}
\end{gathered}
$$

RR $n^{\circ} 4434$ 


$$
\begin{gathered}
+\left(\rho\left(a^{N}-b^{N}\right)-\rho_{1}\left(a^{N+1}-b^{N+1}\right)\right) p(N, N)+\rho \rho_{1}(a-b) p_{0}=0 \\
\rho y \sum_{i=1}^{N-1} v_{i}\left(a^{i}-b^{i}\right) y^{N-1-i}-\rho_{1}(y+q(\rho y-1)) \sum_{i=0}^{N-1} v_{i}\left(a^{i+1}-b^{i+1}\right) y^{N-1-i} \\
\quad+\left(\rho\left(a^{N}-b^{N}\right)-\rho_{1}\left(a^{N+1}-b^{N+1}\right)\right) p(N, N)+\rho \rho_{1}(a-b) p_{0}=0 .
\end{gathered}
$$

By denoting $\cos \varphi=\left(\rho+1-\rho_{2} y\right) /\left(2 \rho_{1}{ }^{1 / 2}\right)$, the roots $a$ and $b$ can be written in the form

$$
a=\frac{\exp (i \varphi)}{\rho_{1}{ }^{1 / 2}}, \quad b=\frac{\exp (-i \varphi)}{\rho_{1}{ }^{1 / 2}} .
$$

Then equation (14) can be rewritten as

$$
\begin{array}{r}
\rho y \sum_{i=1}^{N-1} v_{i} U_{i-1}(t) \frac{y^{N-1-i}}{\rho_{1}{ }^{i / 2}}-\rho_{1}(y+q(\rho y-1)) \sum_{i=0}^{N-1} v_{i} U_{i}(t) \frac{y^{N-1-i}}{\rho_{1}{ }^{(i+1) / 2}} \\
+\left(\rho U_{N-1}(t) \frac{1}{\rho_{1}(N) / 2}-\rho_{1} U_{N}(t) \frac{1}{\rho_{1}(N+1) / 2}\right) p(N, N)+\rho \rho_{1}{ }^{1 / 2} p_{0}=0,
\end{array}
$$

where $t:=\cos \varphi=\left(\rho+1-\rho_{2} y\right) /\left(2 \rho_{1}^{1 / 2}\right)$ and $U_{s}(t)$ are the Chebyshev polynomials of the second kind [3]

$$
U_{s}(\cos \varphi)=\frac{\sin (s+1) \varphi}{\sin \varphi} .
$$

The Taylor series for the function $U_{s}(t)$ with respect to $y$, being actually a polynomial in this case, has the following form

$$
U_{s}(t(y))=\sum_{s=0}^{s} \frac{U_{s}^{(i)}\left(t_{0}\right)}{i !}(-1)^{i} \frac{\rho_{2}^{i} y^{i}}{2^{i} \rho_{1}{ }^{i / 2}}
$$

with $t_{0}=(\rho+1)\left(2 \rho_{1}^{1 / 2}\right)$. By changing the order of summation in the expressions

$$
\begin{aligned}
\sum_{i=1}^{N-1} v_{i} U_{i-1}(t) \frac{y^{N-1-i}}{\rho_{1}{ }^{i / 2}} & =\sum_{l=0}^{N-2} y^{l} \sum_{k=0}^{l} v_{N-1-k} \frac{U_{N-k-2}^{(l-k)}\left(t_{0}\right)\left(-\rho_{2}\right)^{l-k}}{(l-k) ! 2^{l-k} \rho_{1}(N-1-2 k+l) / 2} \\
\sum_{i=0}^{N-1} v_{i} U_{i}(t) \frac{y^{N-1-i}}{\rho_{1}(i+1) / 2} & =\sum_{l=0}^{N-1} y^{l} \sum_{k=0}^{l} v_{N-1-k} \frac{U_{N-k-1}^{(l-k)}\left(t_{0}\right)\left(-\rho_{2}\right)^{l-k}}{(l-k) ! 2^{l-k} \rho_{1}(N-2 k+l) / 2}
\end{aligned}
$$


we rewrite equation (15) as follows:

$$
\begin{gathered}
\rho \sum_{s=1}^{N-1} y^{s} \sum_{k=0}^{s-1} v_{N-1-k} \frac{U_{N-k-2}^{(s-k-1)}\left(t_{0}\right)\left(-\rho_{2}\right)^{s-k-1}}{(s-k-1) ! 2^{s-k-1} \rho_{1}(N-2-2 k+s) / 2} \\
-\rho_{1}(1+\alpha \rho) \sum_{s=1}^{N} y^{s} \sum_{k=0}^{s-1} v_{N-1-k} \frac{U_{N-k-1}^{(s-k-1)}\left(t_{0}\right)\left(-\rho_{2}\right)^{s-k-1}}{(s-k-1) ! 2^{s-k-1} \rho_{1}(N-2 k+s-1) / 2} \\
+\rho_{1} \alpha \sum_{s=0}^{N-1} y^{s} \sum_{k=0}^{s} v_{N-1-k} \frac{U_{N-k-1}^{(s-k)}\left(t_{0}\right)\left(-\rho_{2}\right)^{s-k}}{(s-k) ! 2^{s-k} \rho_{1}(N-2 k+s) / 2} \\
+\left(\rho \sum_{s=0}^{N-1} y^{s} \frac{U_{N-1}^{(s)}\left(t_{0}\right)\left(-\rho_{2}\right)^{s}}{(s) ! 2^{s} \rho_{1}(N+s) / 2}-\rho_{1} \sum_{s=0}^{N} y^{s} \frac{U_{N}^{(s)}\left(t_{0}\right)\left(-\rho_{2}\right)^{s}}{(s) ! 2^{s} \rho_{1}(N+s+1) / 2}\right) p(N, N)+\rho \rho_{1}{ }^{1 / 2} p_{0}=0 .
\end{gathered}
$$

Next we use the relation between the derivatives of the Chebyshev polynomials and Gegenbauer polynomials [3, v.2, p.186]

$$
U_{n}^{(m)}(x)=2^{m} m ! C_{n-m}^{m+1}(x)
$$

to get

$$
\begin{gathered}
\rho \sum_{s=1}^{N-1} y^{s} \sum_{k=0}^{s-1} v_{N-1-k} \frac{C_{N-s-1}^{s-k}\left(t_{0}\right)\left(-\rho_{2}\right)^{s-k-1}}{\rho_{1}(N-2-2 k+s) / 2} \\
-\rho_{1}(1+\alpha \rho) \sum_{s=1}^{N} y^{s} \sum_{k=0}^{s-1} v_{N-1-k} \frac{C_{N-s}^{s-k}\left(t_{0}\right)\left(-\rho_{2}\right)^{s-k-1}}{\rho_{1}(N-2 k+s-1) / 2} \\
+\rho_{1} \alpha \sum_{s=0}^{N-1} y^{s} \sum_{k=0}^{s} v_{N-1-k} \frac{C_{N-s-1}^{s-k+1}\left(t_{0}\right)\left(-\rho_{2}\right)^{s-k}}{\rho_{1}(N-2 k+s) / 2} \\
+\left(\rho \sum_{s=0}^{N-1} y^{s} \frac{C_{N-s-1}^{s+1}\left(t_{0}\right)\left(-\rho_{2}\right)^{s}}{\rho_{1}(N+s) / 2}-\rho_{1} \sum_{s=0}^{N} y^{s} \frac{C_{N-s}^{s+1}\left(t_{0}\right)\left(-\rho_{2}\right)^{s}}{\rho_{1}(N+s+1) / 2}\right) p(N, N)+\rho \rho_{1}{ }^{1 / 2} p_{0}=0 .
\end{gathered}
$$

Collecting the terms with the same power of $y$, we obtain the required system of equations:

- $s=0$

$$
\alpha \rho_{1} C_{N-1}^{1}\left(t_{0}\right) v_{N-1}+\left[\rho C_{N-1}^{1}\left(t_{0}\right)-\rho_{1}{ }^{1 / 2} C_{N}^{1}\left(t_{0}\right)\right] p(N, N)+\rho \rho_{1}{ }^{(N+1) / 2} p_{0}=0
$$

$\mathrm{RR} \mathrm{n}^{\circ} 4434$ 
- $0<s<N$

$$
\begin{gathered}
\rho \sum_{k=0}^{s-1} \frac{C_{N-s-1}^{s-k}\left(t_{0}\right) \rho_{1}{ }^{k+1}}{\left(-\rho_{2}\right)^{k+1}} v_{N-1-k}-\rho_{1}{ }^{3 / 2}(1+\alpha \rho) \sum_{k=0}^{s-1} \frac{C_{N-s}^{s-k}\left(t_{0}\right) \rho_{1}{ }^{k}}{\left(-\rho_{2}\right)^{k+1}} v_{N-1-k} \\
+\alpha \rho_{1} \sum_{k=0}^{s} \frac{C_{N-s-1}^{s-k+1}\left(t_{0}\right) \rho_{1}{ }^{k}}{\left(-\rho_{2}\right)^{k}} v_{N-1-k} \\
+\left[\rho C_{N-s-1}^{s+1}\left(t_{0}\right)-\rho_{1}{ }^{1 / 2} C_{N-s}^{s+1}\left(t_{0}\right)\right] p(N, N)=0
\end{gathered}
$$

- $s=N$

$$
-\rho_{1}^{3 / 2}(1+\alpha \rho) \sum_{k=0}^{N-1} \frac{C_{0}^{N-k}\left(t_{0}\right) \rho_{1}{ }^{k}}{\left(-\rho_{2}\right)^{k+1}} v_{N-1-k}-\rho_{1}{ }^{1 / 2} C_{0}^{N+1}\left(t_{0}\right) p(N, N)=0
$$

or, equivalently,

- $s=0$

$$
\alpha \rho_{1} C_{N-1}^{1}\left(t_{0}\right) v_{N-1}+\left[\rho C_{N-1}^{1}\left(t_{0}\right)-\rho_{1}{ }^{1 / 2} C_{N}^{1}\left(t_{0}\right)\right] p(N, N)+\rho \rho_{1}{ }^{(N+1) / 2} p_{0}=0
$$

- $0<s<N$

$$
\begin{gathered}
\sum_{k=0}^{s-1}\left[\rho \frac{C_{N-s-1}^{s-k}\left(t_{0}\right) \rho_{1}{ }^{k+1}}{\left(-\rho_{2}\right)^{k+1}}-\rho_{1}{ }^{3 / 2}(1+\alpha \rho) \frac{C_{N-s}^{s-k}\left(t_{0}\right) \rho_{1}{ }^{k}}{\left(-\rho_{2}\right)^{k+1}}\right. \\
\left.+\rho_{1} \alpha \frac{C_{N-s-1}^{s-k+1}\left(t_{0}\right) \rho_{1}{ }^{k}}{\left(-\rho_{2}\right)^{k}}\right] v_{N-1-k}+\alpha \frac{C_{N-s-1}^{1}\left(t_{0}\right) \rho_{1}{ }^{s+1}}{\left(-\rho_{2}\right)^{s}} v_{N-1-s} \\
+\left[\rho C_{N-s-1}^{s+1}\left(t_{0}\right)-\rho_{1}{ }^{1 / 2} C_{N-s}^{s+1}\left(t_{0}\right)\right] p(N, N)=0,
\end{gathered}
$$


- $s=N$

$$
-\rho_{1}{ }^{3 / 2}(1+\alpha \rho) \sum_{k=0}^{N-1} \frac{C_{0}^{N-k}\left(t_{0}\right) \rho_{1}{ }^{k}}{\left(-\rho_{2}\right)^{k+1}} v_{N-1-k}-\rho_{1}{ }^{1 / 2} C_{0}^{N+1}\left(t_{0}\right) p(N, N)=0
$$

Finally, to obtain an expression for $A(y)$ in terms of $p(k, N), k=0, \ldots, N$ and Chebyshev polynomials, we again substitute subsequently the roots $a$ and $b$ into (13) and subtract one equation from another

$$
\begin{gathered}
y^{N} \sum_{k=0}^{N-1} \frac{a^{k}-b^{k}}{y^{k}} p(k, N)-\frac{\rho_{1}}{\rho_{2}} \alpha \rho y^{N-1} \sum_{k=0}^{N-1} \frac{a^{k+1}-b^{k+1}}{y^{k}} p(k, N) \\
+\frac{\rho_{1}}{\rho_{2}} \alpha y^{N-1} \sum_{k=0}^{N-1} \frac{a^{k}-b^{k}}{y^{k}} p(k, N)+\left(a^{N}-b^{N}\right) p(N, N)-\frac{\rho_{1}}{\rho_{2}} A(y)(a-b)=0 .
\end{gathered}
$$

As above, taking into account that

$$
\frac{a^{k}-b^{k}}{a-b}=\frac{U_{k-1}(t)}{\rho_{1}^{(k-1) / 2}}
$$

we can express $A(y)$ in terms of $p(k, N), k=0, \ldots, N$ and the Chebyshev polynomials of the second type.

\section{References}

[1] Bondi, A.B., (1989) An analysis of finite capacity queues with priority scheduling and common or reserved waiting areas, Comput. $\& 5$ Ops. Res., 16(3), pp.217233.

[2] Cheng, X., And Akyildiz, F., (1992) A finite buffer two class queue with different scheduling and push-out schemes, IEEE INFOCOM'92, pp. 231-241.

[3] Erdelyi, A., and Bateman, H., (1985) Higher transcendental functions, 2nd ed., v.1,2, Robert E. Krieger Publishing Company.

[4] Jaiswal, N.K., (1968) Priority queues, Academic Press.

$\mathrm{RR} \mathrm{n}^{\circ} 4434$ 
[5] Kapadia, A.S., Kazmi, M.F., And Mitchell, A.C., (1984) Analysis of a finite capacity non-preemptive priority queue, Comput. \&6 Ops. Res., 11(3), pp.337-343.

[6] Kapadia, A.S., Y.K. Chiang, And Kazmi, M.F., (1985) Finite capacity priority queues with potential health applications, Comput. É Ops. Res., 12(4), pp.411-420.

[7] KILKKI, K., (1999) Differentiated services for the Internet, Macmillan Technical Publishing.

[8] Kleinrock, L., (1976) Queueing systems, v.2: Computer Applications, Wiley.

[9] Takagi, H,(1991) Queueing analysis, Vacation and Priority Systems, Part 1. North Holland.

[10] Wagner, D., And Krieger, U.R.,(1999) Analysis of a finite buffer with nonpreemptive priority scheduling, Comm.Stat.-Stochastic Models, 15(2), pp.345365. 


\section{Contents}

1 Introduction $\quad 3$

2 Main results 4

3 Numerical Example and Conclusions $\quad 8$

$\mathrm{RR} \mathrm{n}^{\circ} 4434$ 


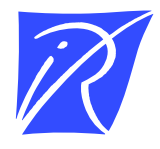

Unité de recherche INRIA Sophia Antipolis 2004, route des Lucioles - BP 93 - 06902 Sophia Antipolis Cedex (France)

Unité de recherche INRIA Lorraine : LORIA, Technopôle de Nancy-Brabois - Campus scientifique 615, rue du Jardin Botanique - BP 101 - 54602 Villers-lès-Nancy Cedex (France)

Unité de recherche INRIA Rennes : IRISA, Campus universitaire de Beaulieu - 35042 Rennes Cedex (France)

Unité de recherche INRIA Rhône-Alpes : 655, avenue de l'Europe - 38330 Montbonnot-St-Martin (France)

Unité de recherche INRIA Rocquencourt : Domaine de Voluceau - Rocquencourt - BP 105 - 78153 Le Chesnay Cedex (France)

Éditeur

INRIA - Domaine de Voluceau - Rocquencourt, BP 105 - 78153 Le Chesnay Cedex (France)

http://www.inria.fr

ISSN 0249-6399 